\title{
Orphan drugs for the treatment of aspergillosis: focus on isavuconazole
}

\author{
Samantha E Jacobs' \\ Vidmantas Petraitis' \\ Catherine B Small' \\ Thomas J Walsh ${ }^{1-3}$ \\ 'Transplantation-Oncology Infectious \\ Diseases Program, Division of \\ Infectious Diseases, Department of \\ Medicine, ${ }^{2}$ Department of Pediatrics, \\ ${ }^{3}$ Department of Microbiology and \\ Immunology, Weill Cornell Medicine, \\ New York, NY, USA
}

This article was published in the following Dove Press journal:

Orphan Drugs: Research and Reviews

3 May 2017

Number of times this article has been viewed

Correspondence: Thomas J Walsh

Weill Cornell Medicine, I 300 York

Avenue, Room A-42I, New York, NY 10065, USA

Tel + I 2127466320

Email thw2003@med.cornell.edu

\begin{abstract}
Invasive aspergillosis (IA) is a particularly devastating manifestation of Aspergillus infection affecting profoundly immunocompromised patients. Voriconazole has been approved as first-line therapy for IA since 2003; however, nonlinear pharmacokinetics, adverse effects, and drug-drug interactions at time hinder its use. Isavuconazole is a new broad-spectrum triazole with potent activity against Aspergillus species. In animal models and clinical trials in humans, isavuconazole has shown comparable efficacy to that of voriconazole in the treatment of IA. Advantages of isavuconazole include a more favorable pharmacokinetic profile and fewer adverse events. This review summarizes the pharmacologic characteristics, in vitro activity, and clinical data supporting the use of isavuconazole as an emerging alternative therapy for IA.

Keywords: isavuconazole, invasive aspergillosis, antifungal therapy, fungal infection
\end{abstract}

\section{Introduction}

Aspergillus species are ubiquitous in the environment and are found in soil, water, food, and air. The usual route of infection is through inhalation of Aspergillus conidia into the lungs. The spectrum of illness varies according to the immune status of the host. Invasive aspergillosis (IA) is a particularly devastating manifestation of Aspergillus infection with mortality rates of $20-40 \%$ depending on the site of infection, underlying immune deficits, and type of therapy. ${ }^{1-3}$ Those at highest risk for IA are profoundly immunocompromised, including patients with chronic granulomatous disease, acute myelogenous leukemia, solid organ and hematopoietic stem cell transplant (HSCT) recipients, patients receiving prolonged corticosteroid therapy, and patients with acquired immunodeficiency syndrome. Less commonly, invasive infection may occur in immunocompetent hosts following local tissue invasion from contaminated central venous catheters or surgical wounds.

The approval of voriconazole, a second-generation triazole, in 2002 was an important therapeutic advance in the treatment of IA. Compared to amphotericin B deoxycholate, patients treated with voriconazole demonstrate higher clinical response rates and decreased mortality. ${ }^{4}$ However, nonlinear pharmacokinetics, drug-drug interactions, side effects, and need for therapeutic drug monitoring at times hinder its use. Isavuconazole is a new broad-spectrum triazole with potent activity against Aspergillus species. It is recommended as an alternative primary therapy for IA syndromes in the 2016 Infectious Diseases Society of America guidelines. ${ }^{5}$ The aim of this review is to summarize the pharmacologic characteristics, in vitro activity, and clinical data supporting the use of isavuconazole for the treatment of IA. 


\section{Role of host immune response}

Defects in the innate and adaptive immune systems may lead to Aspergillus species infection in susceptible hosts. Respiratory epithelial cells act as a physical barrier to invasion by inhaled Aspergillus species by promoting mucociliary clearance. Once the conidia of Aspergillus species are inhaled into the alveoli, the pulmonary alveolar macrophages constitute the first line of innate defense. This is followed by recruitment of peripheral blood monocytes and neutrophils to the site of infection. Neutrophils are a key component of innate immunity as a central cellular component of the inflammatory response. They are the dominant host defense against Aspergillus hyphae, the tissue-invasive form. NADPH oxidase activity in phagocytes generates reactive species of oxygen that facilitate the release of antimicrobial proteases from granules. In addition, pathogen recognition receptors in the host recognize microbial-specific molecules, such as betaglucan in the cell wall of fungi, and activate innate immune responses. Classes of cell-associated and soluble pathogen recognition receptors include toll-like receptors, dectin-1, surfactant proteins $\mathrm{A}$ and $\mathrm{D}$, mannose-binding lectin, and pentraxin-3. The activation of pathogen recognition receptors also promotes maturation of antigen-presenting cells that prime cell-mediated immunity, including helper $\mathrm{T}$ cells and regulatory T cells. ${ }^{6}$

The patient populations at greatest risk for IA are those with qualitative and quantitative defects in neutrophil function. Patients who are profoundly neutropenic lose a critical line of defense against Aspergillus species. Corticosteroids impair several key functions of neutrophils, including phagocytes, oxidative metabolism, phagolysosome formation, release of defensins, and impaired regulation of cytokines and chemokines. Defects in NADPH activity, as seen in patients with chronic granulomatous disease, are associated with recurrent bacterial and fungal infections.

\section{Epidemiology}

The most common Aspergillus species causing invasive infection is A. fumigatus, followed by A. flavus, A. terreus, and more recently, $A$. niger. ${ }^{7,8}$ Recognition of IA depends initially upon the identification of susceptible hosts. The most commonly infected patients are those with a malignancy who develop persistent and profound neutropenia due to chemotherapy or underlying disease and/or are receiving corticosteroids. Indeed, IA remains the most common cause of invasive fungal infection in HSCT recipients despite implementation of anti-mold prophylaxis at many transplant centers and is a leading cause of infection-related mortality in HSCT recipients, as well as those with acute leukemia. ${ }^{9-11}$ Among solid organ transplant recipients, lung transplant patients are particularly at risk for IA. In the Transplant-Associated Infection Surveillance Network, IA accounted for $44 \%$ of invasive fungal infections in this population. ${ }^{2}$ Unique risk factors include a vulnerable bronchial anastomotic site, continuous airway exposure, and transplant disruption of local pulmonary host defenses such as mucociliary clearance. ${ }^{12}$

\section{Clinical manifestations}

The sinopulmonary tract is the most common site of Aspergillus infection. Pulmonary aspergillosis may be classified as acutely invasive, chronic, and allergic. Allergic forms of aspergillosis, such as allergic bronchopulmonary aspergillosis, result from a poorly controlled inflammatory response to hyphae colonizing the sinopulmonary tract. Aspergilloma typifies chronic infection of the lung, such as those involving cavities due to pulmonary tuberculosis, sarcoidosis, bronchiectasis, and cystic fibrosis.

Acute IA of the respiratory tract in immunocompromised patients develops as a bronchopneumonia or as invasive sinusitis. Invasive pulmonary aspergillosis (IPA) may be complicated by pulmonary hemorrhage, hemoptysis, invasion of contiguous structures, or dissemination to extra-thoracic organs. Chronic necrotizing pulmonary aspergillosis is an indolent infection often associated with subtle defects in systemic host defense due to malnutrition, alcoholism, diabetes mellitus, or low-dose corticosteroids. It presents as a chronic refractory bronchopneumonia with fever, weight loss, cough, progressive infiltrates, and evidence of IA on biopsy.

Other target organs for disseminated aspergillosis include the brain, eye, skin, liver, gastrointestinal tract, kidneys, bone, and thyroid. The skin may also be the portal of entry, as reported in cases of intraoperative acquisition and contaminated traumatic or burn wounds. ${ }^{13,14}$

\section{Diagnosis}

Biopsy and culture of tissue is the most definitive means by which to establish a diagnosis of IA. Aspergillus species in tissue forms hyaline angular dichotomously branching septate hyphae. The invasive tissue form has no conidiophores, vesicles, phialides, or conidia. These structures may occasionally be seen, however, in cavitary lesions that communicate directly with the tracheobronchial tree. In patients who are too coagulopathic to undergo biopsy, bronchoalveolar lavage (BAL) fluid from patients with suspected IA should be processed by both clinical microbiology and cytopathology laboratories. BAL fluid may be processed by centrifugation, 
direct examination, and special stains, including fluorescent dyes (Fungi-Fluor ${ }^{\circledR}$, Calcofluor ${ }^{\circledR}$, Blankofluor ${ }^{\circledR}$ ), periodic acid Schiff stain, and Gomori methenamine silver stain.

Galactomannan (GM), a component of fungal cell wall that can be detected by a sandwich-type enzyme-linked immunosorbent assay (ELISA), is used as a diagnostic adjunct for IA. ${ }^{15}$ Depending upon the patient population, sensitivity ranges from $50 \%$ to $95 \%$ and specificity ranges from $87 \%$ to $99 \%$ for the diagnosis of IA. Serial serum GM antigen levels permit therapeutic monitoring and have prognostic implications including clinical response and survival at 12 weeks. ${ }^{16}$ GM testing in BAL fluid has also been evaluated; it demonstrates improved sensitivity compared to serum GM for the diagnosis of IPA. ${ }^{17}$

$(1 \rightarrow 3)-\beta$-D-glucan is a cell-wall-derived biomarker for detection of invasive fungal infections, including IA. However, the detection of $(1 \rightarrow 3)-\beta$-D-glucan in serum is not specific for IA and warrants further evaluation such as BAL for immunocompromised patients with pulmonary infiltrates.

Real-time polymerase chain reaction (PCR) for the diagnosis of invasive fungal infection has been studied most extensively with Aspergillus species. Most studies test for a pan-Aspergillus PCR that targets ribosomal RNA common to all Aspergillus species; however, primers that are used vary among laboratories, raising issues of standardization. Clinical reports of sensitivities and specificities range from $43 \%$ to $100 \%$ and $64 \%$ to $100 \%$, respectively. ${ }^{18}$ Studies comparing the diagnostic performance of PCR and GM assays for Aspergillus species show similar performance in both serum and BAL fluid. ${ }^{19}$

Of note, the sensitivity of GM, $(1 \rightarrow 3)-\beta$-D-glucan, and $\mathrm{PCR}$ is considerably reduced in patients receiving antifungal prophylaxis, such that routine screening using these biomarkers is not recommended in that population.

\section{Treatment}

Since 2002, voriconazole has been licensed for the primary treatment of IA in most patients. ${ }^{20}$ In the largest randomized controlled trial of therapy for IA, voriconazole was associated with significantly improved survival (71\% versus $58 \%$ ) compared to amphotericin B deoxycholate. ${ }^{21}$ Liposomal amphotericin B has also been studied for primary therapy of IA and is associated with 12-week survival rates of $72 \%$ and $59 \%$ at doses of $3 \mathrm{mg} / \mathrm{kg}$ per day and $10 \mathrm{mg} / \mathrm{kg}$ per day, respectively. ${ }^{22}$ Liposomal amphotericin B may also be useful as primary therapy for patients with preexisting liver disease or in those with ultrarapid metabolizing genotypes $^{23}$ and suspected mixed infection with mucormycosis. Recently, the role of combination therapy with voriconazole and anidulafungin in the treatment of IA as primary or salvage therapy is suggested by preclinical data and by a recent prospective, controlled clinical trial. ${ }^{24,25}$

The development of voriconazole represented a major advance in the therapy of aspergillosis. However, the drug's side effect profile, drug-drug interactions, and need for therapeutic drug monitoring pose management challenges in immunocompromised patients with multiple comorbid conditions. Isavuconazole is the newest triazole to be approved for the treatment of IA and the first triazole to be approved as primary therapy for mucormycosis. ${ }^{26}$ Robust preclinical data support its efficacy as comparable or greater than that of other antifungal agents in the treatment of IA. Moreover, in phase 2 and 3 clinical trials, isavuconazole is safer and better tolerated than voriconazole, thus offering an emerging alternative. The following sections review the pharmacology of isavuconazole and the in vitro, in vivo, and clinical data for its use in the treatment of IA.

\section{Isavuconazole Chemical structure and mechanism of action}

Isavuconazole is administered as a prodrug, known as isavuconazonium sulfate (Figure 1), which rapidly releases the parent molecule, isavuconazole (Figure 2), in the presence of serum esterases. By comparison, voriconazole is administered as a triazole solubilized in sulfobutylether cyclodextrin (Figures 3 and 4). The active molecules, voriconazole and isavuconazole, differ structurally in several respects. Both molecules share an isopropyl alcohol core, a C-1 triazolyl moiety, C-2-di-fluorophenyl substitution, and a 3-alpha-methyl group. However, located on the C-3-atom, voriconazole has a fluoropyrimidinyl group (Figure 3), while isavuconazole has a thiazolyl-benzonitrile substitution (Figure 2).

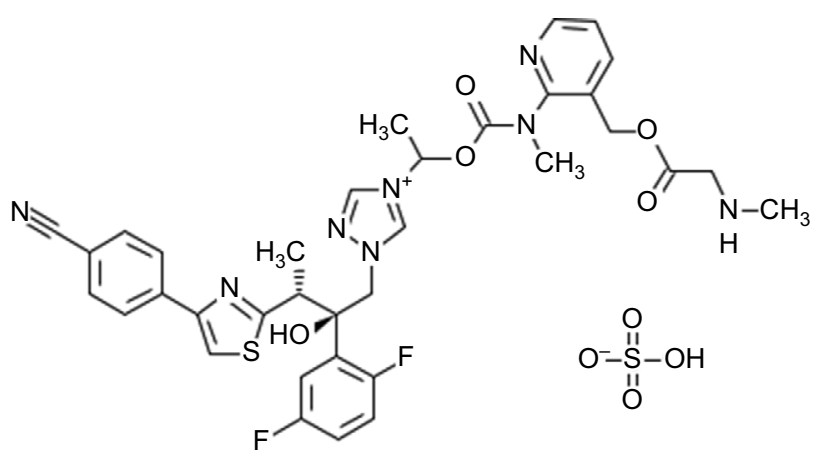

Figure I Chemical structure of isavuconazonium sulfate. 


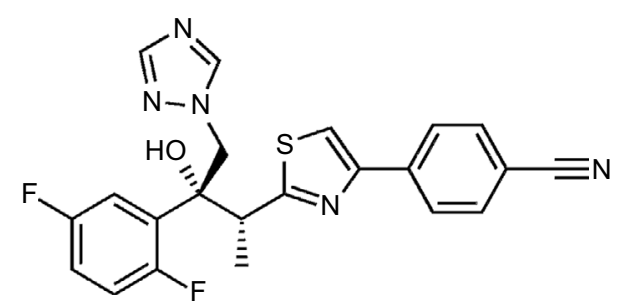

Figure 2 Chemical structure of isavuconazole.<smiles>C[C@@H](c1ncncc1F)[C@](O)(Cn1cncn1)c1ccc(F)cc1F</smiles>

Figure 3 Chemical structure of voriconazole.

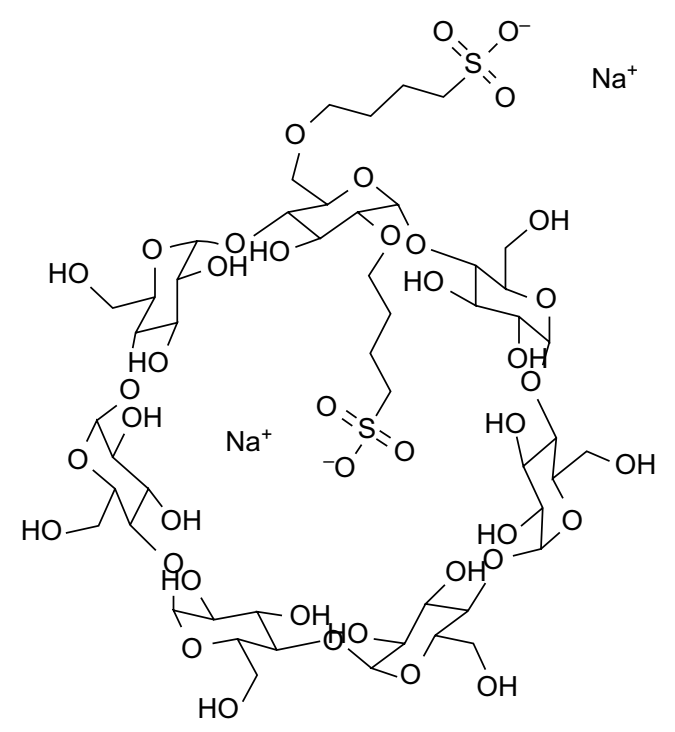

Figure 4 Chemical structure of sulfobutylether cyclodextrin.

Similar to other azoles, isavuconazole prevents fungal cell wall synthesis via inhibition of lanosterol $14 \alpha$-demethylase. This cytochrome P450 enzyme catalyzes demethylation of lanosterol, thereby forming ergosterol, the predominant sterol in the fungal cell membrane. ${ }^{27}$ The thiazolyl cyanophenyl moiety of the active isavuconazole molecule allows greater avidity of isavuconazole for the binding pocket in the fungal cytochrome $\mathrm{P} 450$ (CYP) 51 protein, conferring broader antifungal spectrum even to pathogens resistant to other azoles.

\section{Spectrum of activity}

Isavuconazole has broad in vitro activity against many yeasts and molds including Aspergillus species, Mucorales, Fusarium species, and dematiaceous molds. ${ }^{28}$

\section{Pharmacokinetics}

Table 1 compares the pharmacokinetic profiles of isavuconazole versus voriconazole. The pharmacokinetic profile of isavuconazole is well described in healthy volunteers. ${ }^{29,30}$ Isavuconazole is more than $99 \%$ protein bound in serum, has a large volume of distribution (approximately $450 \mathrm{~L}$ ), and displays an elimination half-life of $\sim 80-130$ hours. ${ }^{27}$ To achieve rapid steady state concentrations, the drug is administered as a loading dose of $200 \mathrm{mg}$ every 8 hours for six doses, followed by $200 \mathrm{mg}$ once daily. Isavuconazole follows linear dose proportionality of area under the curves (AUCs) within the dosage ranges studied. The drug is available in intravenous (IV) and oral formulations. The oral bioavailability is $98 \%$, and the maximum plasma concentration $\left(C_{\max }\right)(2-2.5 \mu \mathrm{g} / \mathrm{mL})$ is reached in $1-3$ hours. ${ }^{27}$

Hepatic metabolism is the primary mode of elimination. The isavuconazole molecule is eliminated largely unchanged by the liver. Minor metabolites are produced by CYP3A4 and CYP3A5 as the predominant enzymes involved in phase 1 metabolism, followed by modification by uridine diphosphate glucuronosyltransferase (UGT) and excretion in feces and bile. Subjects with mild-to-moderate liver disease receiving a single dose of isavuconazole demonstrate decreased clearance of isavuconazole as indicated by increased serum concentrations, increased mean half-life, and increased total systemic exposure measured as $\mathrm{AUC}_{0 \rightarrow \infty}{ }^{31}$ Desai et al ${ }^{32}$ used a modeling procedure combining data from two studies to determine the pharmacokinetics of isavuconazole in patients with mild-to-moderate hepatic impairment receiving the recommended clinical dosage. A less than twofold increase in the plasma isavuconazole trough concentration was calculated.

Table I Comparison of the pharmacokinetic profiles of isavuconazole and voriconazole

\begin{tabular}{|c|c|c|}
\hline Parameters & $\begin{array}{l}\text { Isavuconazole } \\
(200 \mathrm{mg} / \text { day })\end{array}$ & $\begin{array}{l}\text { Voriconazole } \\
(4 \mathrm{mg} / \mathrm{kg} \text { twice/day })\end{array}$ \\
\hline Available formulations & Oral or IV & Oral or IV \\
\hline Oral bioavailability & $98 \%$ & $96 \%$ \\
\hline Food effect & Absent & $\begin{array}{l}C_{\max } \text { and } A U C \text { reduced } \\
\text { by high-fat meals }\end{array}$ \\
\hline$C_{\max }$ at steady state & $4 \mu \mathrm{g} / \mathrm{mL}$ & $5.4 \mu \mathrm{g} / \mathrm{mL}$ \\
\hline Elimination half-life & 130 hours & Dose-dependent ${ }^{a}$ \\
\hline Protein binding & $>99 \%$ & $58 \%$ \\
\hline Volume of distribution & $450 \mathrm{~L}$ & $4.6 \mathrm{~L} / \mathrm{kg}$ \\
\hline CSF penetration & No data available & 250\% CSF:plasma \\
\hline Metabolism & Hepatic & Hepatic \\
\hline Elimination & $\begin{array}{l}45 \% \text { feces, } 45 \% \text { urine } \\
\text { (as inactive metabolites) }\end{array}$ & $\begin{array}{l}>80 \% \text { urine (as } \\
\text { inactive metabolites) }\end{array}$ \\
\hline Dose proportionality & Linear & Nonlinear \\
\hline
\end{tabular}

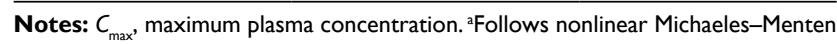
saturation kinetics. Data from Rybak et a ${ }^{27}$ and Astellas. ${ }^{35}$

Abbreviations: AUC, area under the curve; CSF, cerebrospinal fluid; IV, intravenous. 
At this time, no dosage adjustment is recommended in liver dysfunction. Less than $1 \%$ of isavuconazole is excreted unchanged in urine, and no renal dosage adjustments are necessary.

The safety and pharmacokinetics of isavuconazole administered as antifungal prophylaxis in patients with prolonged neutropenia have been evaluated in one study. ${ }^{33}$ This openlabel, sequential cohort, phase 2 study assigned 24 patients with acute myelogenous leukemia receiving induction or subsequent chemotherapy to receive low-dose (12 patients) or high-dose (12 patients) IV isavuconazole for a maximum of 28 days. The low-dose cohort received three loading doses of IV isavuconazole at $400 \mathrm{mg}, 200 \mathrm{mg}$, and $200 \mathrm{mg}$ every 8 hours on day 1 , followed by further loading doses of $200 \mathrm{mg}$ twice daily on day 2 , and then a once-daily maintenance dose of $200 \mathrm{mg}$ from day 3 to the end of treatment. The high-dose cohort received doses of isavuconazole that were twofold higher, i.e., $800 \mathrm{mg} / 400 \mathrm{mg} / 400 \mathrm{mg}$ on day $1,400 \mathrm{mg}$ twice daily on day 2, and $400 \mathrm{mg}$ once daily thereafter. A total of 21 and 18 patients were evaluable for pharmacokinetic analyses on days 1 and 7, respectively. At 12 hours after the start of treatment, the mean plasma isavuconazole concentration was $1.5 \mu \mathrm{g} / \mathrm{mL}$ and $2.5 \mu \mathrm{g} / \mathrm{mL}$ in the low- and high-dose cohorts, respectively. The $C_{\max }$ and the area under the plasma concentration-time curve from time 0 hour to 24 hours after the initiation of isavuconazole administration $\left(\mathrm{AUC}_{0-24}\right)$ on day 7 were $3.6 \mu \mathrm{g} / \mathrm{mL}$ and $60.1 \mu \mathrm{g} \cdot \mathrm{h} / \mathrm{mL}$ in the low-dose cohort, respectively, and $8.0 \mu \mathrm{g} / \mathrm{mL}$ and $113.1 \mu \mathrm{g} \cdot \mathrm{h} / \mathrm{mL}$ in the high-dose cohort, respectively. At day 7 , the interpatient variability for $C_{\max }$ and $\mathrm{AUC}_{0-24}$ was low. Of note, the $95 \%$ confidence intervals of the ratios of isavuconazole dosenormalized geometric mean $C_{\max }$ and $\mathrm{AUC}_{0-24}$ values were slightly outside the normal acceptance range.

\section{Tissue penetration}

The concentration of isavuconazole in epithelial lining fluid (ELF) correlates well with that obtained in plasma but is lower, including the $C_{\max }$ of isavuconazole. In one study, the penetration of isavuconazole in ELF compared to plasma based on total drug was between $35.8 \%$ and $72.5 \% .^{34}$ Studies of penetration of isavuconazole into cerebrospinal fluid (CSF), brain tissue, and vitreous are limited and warrant further quantitative evaluation.

\section{Drug interactions}

Triazole antifungal agents inhibit CYP enzymes, although the degree to which they inhibit different CYP families varies according to compound. Isavuconazole is both a sensitive substrate of and a mild-to-moderate inhibitor of CYP3A4. Potential drug-drug interactions are shown in Figure 5. Rifampin, a potent $3 \mathrm{~A} 4$ inducer, decreases isavuconazole plasma AUC by 40-fold. Ketoconazole, a strong inhibitor of CYP enzymes, increases the isavuconazole plasma AUC by fivefold. Up to a twofold increase is seen in midazolam plasma AUC and a 1.84-fold increase in sirolimus plasma AUC when these drugs are administered concurrently with isavuconazole. In drug-drug interaction studies, isavuconazole did not affect the pharmacokinetics of substrates

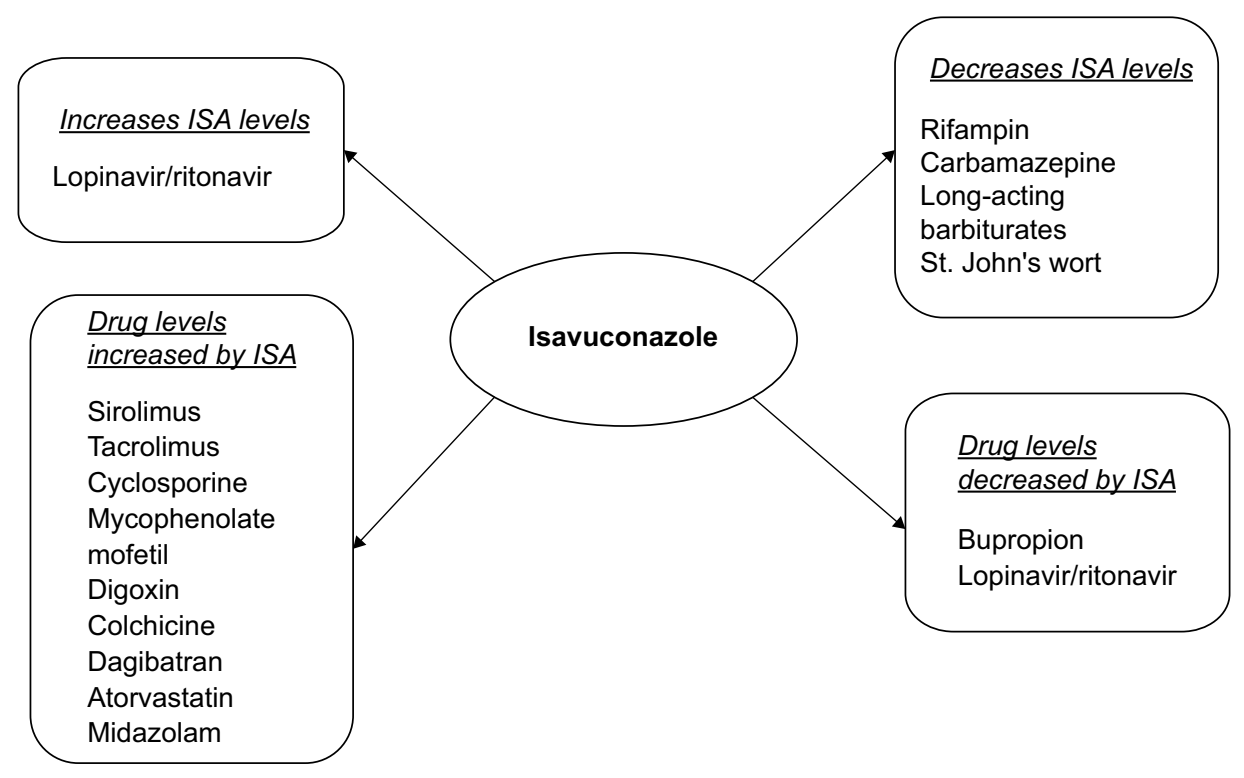

Figure 5 Drug-drug interactions with ISA.

Note: Data from Miceli and Kauffman. ${ }^{26}$

Abbreviation: ISA, isavuconazole. 
of CYP1A2, CYP2C8, CYP2C9, CYP2C19, or CYP2D6, although it did have mild inhibitory effects on $\mathrm{P}$-glycoprotein, substrates of UGT and CYP2B6. ${ }^{35}$

In the patient population most likely to receive isavuconazole for treatment and prophylaxis, potentially clinically relevant drug-drug interactions include rifampin, sirolimus, tacrolimus, cyclosporine, mycophenolate mofetil, cyclophosphamide, and vincristine. Groll et $\mathrm{al}^{36}$ reported several phase 1 drug-drug interaction studies in healthy adults receiving clinical doses of oral isavuconazole $(200 \mathrm{mg}$ three times daily for 2 days; $200 \mathrm{mg}$ once daily thereafter). These studies demonstrated the following increases in mean whole blood or plasma $\mathrm{AUC}_{0-\infty}$ : tacrolimus, $125 \%$; sirolimus, $84 \%$; cyclosporine, 29\%; and mycophenolic acid, 35\%. Mean $C_{\max }$ values of tacrolimus, sirolimus, and cyclosporine were $42 \%, 65 \%$, and $6 \%$ higher, respectively; mean $C_{\max }$ of mycophenolic acid was $11 \%$ lower. There was little change to the plasma AUC of prednisolone when prednisone and isavuconazole were given together. Overall, the degree of interaction between isavuconazole and these immunosuppressive agents was less than that which has been previously reported with other triazoles including voriconazole. However, attention to therapeutic drug monitoring of these immunosuppressive agents and possible dose adjustments are likely to be necessary for cyclosporine, sirolimus, and tacrolimus in patients receiving concomitant isavuconazole to ensure adequate concentrations and to avoid adverse toxicokinetic effects. Also of note, the CYP2C19 gene polymorphisms, which may necessitate a change in antifungal agent or dosage adjustment for voriconazole, ${ }^{23}$ are not observed in isavuconazole metabolism.

\section{In vitro activity against Aspergillus species}

Isavuconazole demonstrates antifungal activity against a wide range of Aspergillus species. In studies using Clinical and Laboratory Standards Institute (CLSI) methodology, the MIC50 and MIC90 for the two most common Aspergillus species, A. fumigatus and A. flavus, ranged $0.5-2 \mu \mathrm{g} / \mathrm{mL}$ and $1-2 \mu \mathrm{g} / \mathrm{mL}$ for $A$. fumigatus and $0.5-2 \mu \mathrm{g} / \mathrm{mL}$ and $1-4 \mu \mathrm{g} / \mathrm{mL}$ for A. flavus (Table 2). ${ }^{37-40}$ Higher minimum inhibitory concentration (MIC) values have consistently been observed with A. niger. ${ }^{37,38,40,41}$ When obtained by using the methodology set by the European Committee on Antimicrobial Susceptibility Testing (EUCAST), the MIC50 and MIC90 of isavuconazole against Aspergillus species appear similar. ${ }^{39,42,43}$

The clinical significance of MIC variability according to Aspergillus species is not yet clear. However, it is important to identify organisms to the species level in the clinical microbiology laboratory because species-specific epidemiologic cutoff values (ECVs) will aid identification
Table 2 In vitro activity of isavuconazole against different Aspergillus species ${ }^{\mathrm{a}, \mathrm{b}}$

\begin{tabular}{llllll}
\hline Species & $\mathbf{N}$ & $\begin{array}{l}\text { MIC50 } \\
(\mu \mathrm{g} / \mathbf{m L})\end{array}$ & $\begin{array}{l}\text { MIC90 } \\
(\boldsymbol{\mu g} / \mathbf{m L})\end{array}$ & $\begin{array}{l}\text { MIC range } \\
(\mu \mathrm{g} / \mathbf{m L})\end{array}$ & References \\
\hline A. fumigatus & 14 & 2 & 2 & $\mathrm{I}-4$ & 37 \\
& 602 & $\mathrm{I}$ & $\mathrm{I}$ & $0.125-4$ & 38 \\
& 62 & 0.5 & 2 & $0.125-2$ & 40 \\
A. flavus & 12 & 2 & 4 & $\mathrm{I}-4$ & 37 \\
& 34 & $\mathrm{I}$ & $\mathrm{I}$ & $0.25-2$ & 38 \\
& 187 & - & - & $0.125-2$ & 39 \\
& 20 & 0.5 & $\mathrm{I}$ & $0.5-2$ & 40 \\
A. terreus & 19 & $\mathrm{I}$ & 2 & $0.5-4$ & 37 \\
& 25 & $\mathrm{I}$ & $\mathrm{I}$ & $0.125-1$ & 38 \\
& 18 & 0.5 & 0.5 & $0.25-0.5$ & 40 \\
A. niger & 2 & - & - & $2-4$ & 37 \\
& 32 & $\mathrm{I}$ & 2 & $0.25-4$ & 38 \\
& 18 & 0.5 & 2 & $0.25-2$ & 40 \\
\hline
\end{tabular}

Notes: ${ }^{\mathrm{D} D a t a}$ were obtained using the CLSI methodology. ${ }^{\mathrm{b}}$ Cells were marked with a dash ("-") when MIC50 and MIC90 data were not available.

Abbreviation: CLSI, Clinical and Laboratory Standards Institute.

of resistant isolates. Espinel-Ingroff et $\mathrm{al}^{44}$ defined isavuconazole ECVs for wild-type Aspergillus species using MIC data from laboratories in Europe, India, Mexico, and the USA. MICs were determined by the CLSI M38-A2 broth microdilution method. ECVs were $1 \mu \mathrm{g} / \mathrm{mL}$ for $A$. fumigatus species complex, $1 \mu \mathrm{g} / \mathrm{mL}$ for $A$. flavus species complex, $0.25 \mu \mathrm{g} / \mathrm{mL}$ for $A$. nidulans species complex, $4 \mu \mathrm{g} / \mathrm{mL}$ for A. niger species complex, $1 \mu \mathrm{g} / \mathrm{mL}$ for $A$. terreus species complex, and $1 \mu \mathrm{g} / \mathrm{mL}$ for $A$. versicolor species complex. The EUCAST described similar ECVs for Aspergillus species and recently determined interpretive break points for isavuconazole and Aspergillus species. ${ }^{45}$ The break point is $1 \mu \mathrm{g} / \mathrm{mL}$ for $A$. fumigatus and $A$. terreus and $0.25 \mu \mathrm{g} / \mathrm{mL}$ for A. nidulans. The EUCAST concluded that there is insufficient evidence to establish interpretive break points for other Aspergillus species. CLSI has not established clinical break points for isavuconazole and Aspergillus species.

Isavuconazole appears to have resistance patterns similar to those of voriconazole. A. fumigatus isolates with molecularly characterized cyp 51A alterations L98H, G138C, Y431C, G434C, and G448S showed elevated MICs to all triazoles, including isavuconazole. ${ }^{46}$ The greatest isavuconazole MIC elevations are observed in the TR34/L98H mutants. ${ }^{43,46}$ In contrast, the isavuconazole MICs of the majority of G54 mutants were within the wild-type range. ${ }^{43,46}$ Isolates with G54 alterations tend to demonstrate itraconazole and posaconazole resistance while maintaining voriconazole susceptibility. Therefore, in clinical practice, isavuconazole should be avoided for the treatment of infections with Aspergillus species with elevated voriconazole MICs. Furthermore, MIC testing for isavuconazole should be performed on cultures of infecting organisms when available. 


\section{Animal models}

Preclinical animal model pharmacokinetic/pharmacodynamic investigations are important for defining antifungal efficacy, safety, and dosage optimization. Animal models provide a framework for predicting drug exposure and its relationship to clinical outcome. In addition, they allow examination of the susceptibility break points in an era where drug resistance is increasingly common. The efficacy of isavuconazole has been evaluated in experimental models of disseminated candidiasis and aspergillosis. Lepak et $\mathrm{al}^{47}$ examined the pharmacodynamics of isavuconazole in a murine model of IPA that included wild-type and cyp 51 mutant isolates of $A$. fumigatus. The investigators demonstrated that the isavuconazole pharmacodynamic index AUC/ MIC ratio (median free-drug value of 5.0) correlates well with treatment outcome. An MIC of $0.5-1 \mu \mathrm{g} / \mathrm{mL}$ was a strong predictor of success regardless of the presence or absence of a cyp 51 mutation. Furthermore, in a neutropenic murine model of disseminated A. flavus, isavuconazole treatment led to decreased fungal tissue burden and improved survival similar to itraconazole and voriconazole. ${ }^{48}$

A rabbit model of experimental IPA has been used to further define the pharmacokinetics and pharmacodynamics of isavuconazole. ${ }^{49,50}$ Persistently, neutropenic rabbits treated with isavuconazole at $40 \mathrm{mg} / \mathrm{kg}$ per day and $60 \mathrm{mg} / \mathrm{kg}$ per day demonstrated significant dose-dependent reduction in residual fungal burden, decreased pulmonary injury, prolonged survival, lower GM index in serum and BAL fluid, and lower serum $(1 \rightarrow 3)-\beta$-D-glucan levels..$^{50}$ Using mathematical modeling, Kovanda et $\mathrm{al}^{49}$ evaluated the exposure-response relationship of this model of experimental IPA using reduction in GM index as a marker of disease clearance. This bridging analysis using Monte Carlo simulation demonstrated a strong concordance with the clinical trial and the robustness of the rabbit model of IPA to predict patient outcomes.

\section{Combination therapy}

Response to treatment with a single antifungal agent is often unsuccessful, as acquired resistance and breakthrough infections have been reported among patients with long-term exposure to a single antifungal drug class. Combination antifungal therapy is a strategy to improve antimicrobial activity and clinical outcomes.

In vitro combination studies have found that isavuconazole and micafungin are synergistically active against $A$. fumigatus, A. flavus, and $A$. terreus. In contrast, the interaction between the combination of isavuconazole and amphotericin $\mathrm{B}$ deoxycholate was antagonistic in A. fumigatus and A. flavus and indifferent in $A$. terreus. ${ }^{51}$

Combination therapy has been studied in vivo in persistently neutropenic rabbits with experimental IPA (A. fumigatus). Compared to rabbits treated with isavuconazole monotherapy, Petraitis et al ${ }^{52}$ demonstrated that rabbits treated with isavuconazole plus micafungin demonstrated synergistic interaction resulting in significantly lower serum GM index, serum ( $1 \rightarrow 3)-\beta$-D-glucan levels, and mortality. In addition, synergistic interaction of combinations of isavuconazole $20 \mathrm{mg} / \mathrm{kg}$ per day or $40 \mathrm{mg} / \mathrm{kg}$ per day plus micafungin was observed in the reduction of organism-mediated pulmonary injury, resulting in significantly lower lung weights and pulmonary infarct scores. Clinical studies are needed to better understand the role of combination therapy in the treatment of IA in human subjects.

\section{Clinical trials}

\section{Invasive aspergillosis}

Extensive preclinical data have established that isavuconazole has potent in vitro and in vivo activity against most Aspergillus species. These data served as a basis for the SECURE clinical trial, a multicenter, randomized, double-blind, non-inferiority trial of isavuconazole versus voriconazole for the treatment of invasive fungal infections due to Aspergillus species and other filamentous fungi. ${ }^{53}$ Adult patients with proven, probable, or possible invasive fungal infection according to established criteria ${ }^{54}$ were randomized in a 1:1 ratio to treatment with isavuconazole (200 mg IV three times per day for six doses followed by $200 \mathrm{mg}$ IV or orally daily thereafter) or voriconazole $(6 \mathrm{mg} / \mathrm{kg}$ IV twice daily for two doses followed by $4 \mathrm{mg} / \mathrm{kg}$ IV twice daily or $200 \mathrm{mg}$ orally twice daily thereafter). The primary outcome measure of the trial was day 42 all-cause mortality in the intention-to-treat (ITT) arm using a 10\% non-inferiority margin. Of note, patients were excluded if they had chronic pulmonary aspergillosis or allergic bronchopulmonary aspergillosis, if they had received a mold-active triazole for $\geq 4$ days in the 1 week prior to starting the study drug, or if they had a creatinine clearance of $<50 \mathrm{~mL} / \mathrm{min}$.

Hematological malignancies were the most common underlying condition (84\%); 65\% were neutropenic, and $20 \%$ had received an allogeneic hematopoietic cell transplant. The median durations of treatment were 45 days and 47 days for patients receiving isavuconazole and voriconazole, respectively. All-cause mortality through day 42 in the ITT population of 516 subjects was $18.6 \%$ 
and $20.2 \%$ in the isavuconazole and voriconazole treatment groups, respectively, meeting the primary objective of non-inferiority. Isavuconazole was also non-inferior to voriconazole in the prespecified analysis of 231 patients with proven or probable IA in which all-cause mortality on day 42 was 19\% in isavuconazole-treated patients and $22 \%$ in voriconazole-treated patients.

Of note, the relationship between clinical response and Aspergillus MIC was evaluated among those patients who had Aspergillus species cultured at baseline. ${ }^{35}$ Among isavuconazole-treated patients, using CLSI methods, isavuconazole demonstrated MIC50 and MIC90 values against 51 baseline Aspergillus species isolates of $1 \mu \mathrm{g} / \mathrm{mL}$ and $4 \mu \mathrm{g} / \mathrm{mL}$, respectively, with MICs ranging from $0.25 \mu \mathrm{g} / \mathrm{mL}$ to $32 \mu \mathrm{g} /$ $\mathrm{mL}$. Voriconazole MIC values for these isolates were similar (MIC50: $1 \mu \mathrm{g} / \mathrm{mL}$; MIC90: $2 \mu \mathrm{g} / \mathrm{mL}$; range: 0.12-32 $\mu \mathrm{g} / \mathrm{mL}$ ). Among voriconazole-treated patients, voriconazole MIC50 and MIC90 values against 25 baseline Aspergillus isolates of $1 \mu \mathrm{g} / \mathrm{mL}$ and $2 \mu \mathrm{g} / \mathrm{mL}$, respectively, with MICs ranging from $0.25 \mu \mathrm{g} / \mathrm{mL}$ to $2 \mu \mathrm{g} / \mathrm{mL}$. Isavuconazole MIC values for these isolates were similar (MIC50: $1 \mu \mathrm{g} / \mathrm{mL}$; MIC90: $2 \mu \mathrm{g} / \mathrm{mL}$; range: $0.25-4 \mu \mathrm{g} / \mathrm{mL}$ ). Overall response at the end of treatment was favorable at a range of MIC values in both the isavuconazole and voriconazole treatment groups. There was no observed relationship between outcomes and MIC.

Treatment with isavuconazole was generally well-tolerated, and drug-related treatment-emergent adverse events (TEAEs) occurred less frequently in the isavuconazole versus voriconazole treatment groups. Further discussion of isavuconazole safety is provided in the following section.

\section{Adverse effects}

Treatment with isavuconazole is generally well tolerated. The relatively greater safety and tolerability of isavuconazole compared to voriconazole is a key distinguishing feature of the drug. Approximately 1700 total patients have received isavuconazole in phase 1, 2, and 3 studies. In the VITAL trial of isavuconazole for the treatment of mucormycosis ${ }^{55}$ and in the SECURE trial the most common adverse events were nausea, diarrhea, vomiting, pyrexia, constipation, and hypokalemia. In the SECURE trial, significantly fewer drug-related TEAEs occurred in patients treated with isavuconazole $(42 \%)$ versus voriconazole $(60 \%)(p<0.001) .{ }^{53}$ In particular, fewer adverse events occurred in the following system-organ classes in patients receiving isavuconazole versus voriconazole: hepatobiliary disorders ( $9 \%$ versus $16 \%$ ), eye disorders (15\% versus
$27 \%)$, and skin and subcutaneous tissue disorders $(33 \%$ versus $42 \%$ ). Permanent drug discontinuation due to TEAEs was $14 \%$ and $23 \%$ in patients taking isavuconazole and voriconazole, respectively.

Most triazole antifungal agents are associated with QT prolongation. Notably, in the SECURE and VITAL studies, isavuconazole caused dose-dependent QTc shortening of up to $13 \mathrm{~ms}$ at the Cmax of the proposed $200 \mathrm{mg}$ maintenance dose. ${ }^{35}$ The clinical significance of this observed QT shortening is unknown. No ventricular arrhythmias were observed, and no medical interventions were required. However, isavuconazole is currently contraindicated in patients with familial short QT syndrome.

\section{Future directions}

A growing body of in vitro, animal, and human data continues to support the clinical use of isavuconazole for the treatment of infections due to Aspergillus species and other fungi. Additional research is needed, however, in several key areas. Isavuconazole has not been studied in patients with chronic pulmonary and allergic forms of aspergillosis or in patients with central nervous system and musculoskeletal aspergillosis. Pediatric data, including pharmacokinetic, pharmacodynamics, safety, and efficacy studies, are needed. Drug-drug interaction studies with other immunosuppressive agents such as vincristine are crucial in patients receiving chemotherapy. Finally, initiatives to understand the role of isavuconazole in antifungal prophylaxis in immunocompromised hosts are being developed.

\section{Conclusion}

As shown in a large randomized controlled clinical trial and supported by preclinical data, isavuconazole is at least as effective as voriconazole for the treatment of IA. Advantages to isavuconazole include its predictable, linear pharmacokinetics, high prodrug water solubility (such that cyclodextrin is not needed), and fewer adverse effects. Clinical experience with isavuconazole remains limited, and therefore, voriconazole remains the first-line therapy for aspergillosis syndromes. However, isavuconazole is an emerging alternative, particularly in patients intolerant of voriconazole.

\section{Acknowledgments}

TJW is a Scholar of the Save Our Sick Children Foundation and a Scholar of the Sharp Family Foundation in Pediatric Emerging Infectious Diseases. This work was funded in part by the Save Our Sick Children Foundation (to TJW). 


\section{Disclosure}

TJW has received research grants for experimental and clinical antimicrobial pharmacotherapeutics from Astellas, Cubist, Novartis, Pfizer, Allergan, and Theravance. He also has served as consultant to Astellas, ContraFect, Cubist, Drais, iCo, Novartis, Pfizer, Methylgene, SigmaTau, and Trius. The other authors report no conflicts of interest in this work.

\section{References}

1. Neofytos D, Horn D, Anaissie E, et al. Epidemiology and outcome of invasive fungal infection in adult hematopoietic stem cell transplant recipients: analysis of multicenter prospective antifungal therapy (PATH) alliance registry. Clin Infect Dis. 2009;48(3):265-273.

2. Pappas PG, Alexander BD, Andes DR, et al. Invasive fungal infections among organ transplant recipients: results of the transplant-associated infection surveillance network (TRANSNET). Clin Infect Dis. 2010; 50(8):1101-1111.

3. Pagano L, Caira M, Candoni A, et al. The epidemiology of fungal infections in patients with hematologic malignancies: The SEIFEM-2004 study. Haematologica. 2006;91(8):1068-1075.

4. Herbrecht R, Denning DW, Patterson TF, et al; Invasive Fungal Infections Group of the European Organisation for Research and Treatment of Cancer and the Global Aspergillus Study Group. Voriconazole versus amphotericin B for primary therapy of invasive aspergillosis. $N$ Engl J Med. 2002;347(6):408-415.

5. Patterson TF, Thompson GR 3rd, Denning DW, et al. Practice guidelines for the diagnosis and management of aspergillosis : 2016 update by the Infectious Diseases Society of America. Clin Infect Dis. 2016;63:1-60.

6. Segal BH. Aspergillosis. N Engl J Med. 2009;360(18):1870-1884.

7. Morgan J, Wannemuehler KA, Marr KA, et al. Incidence of invasive aspergillosis following hematopoietic stem cell and solid organ transplantation: interim results of a prospective multicenter surveillance program. Med Mycol. 2005;43(suppl 1):49-58.

8. Steinbach WJ, Marr KA, Anaissie EJ, et al. Clinical epidemiology of 960 patients with invasive aspergillosis from the PATH Alliance registry. $J$ Infect. 2012;65(5):453-464.

9. Corzo-Leon DE, Satlin MJ, Soave R, et al. Epidemiology and outcomes of invasive fungal infections in allogeneic haematopoietic stem cell transplant recipients in the era of antifungal prophylaxis: a single-centre study with focus on emerging pathogens. Mycoses. 2015;58(6):325-336.

10. Girmenia C, Raiola AM, Piciocchi A, et al. Incidence and outcome of invasive fungal diseases after allogeneic stem cell transplantation: a prospective study of the Gruppo Italiano Trapianto Midollo Osseo (GITMO). Biol Blood Marrow Transplant. 2014;20(6):872-880.

11. Nucci M, Garnica M, Gloria AB, et al. Invasive fungal diseases in haematopoietic cell transplant recipients and in patients with acute myeloid leukaemia or myelodysplasia in Brazil. Clin Microbiol Infect. 2013;19(8):745-751.

12. Margalit A, Kavanagh K. The innate immune response to Aspergillus fumigatus at the alveolar surface. FEMS Microbiol Rev. 2015;39(5):670-687.

13. Steinbach WJ, Benjamin DK, Kontoyiannis DP, et al. Infections due to Aspergillus terreus: a multicenter retrospective analysis of 83 cases. Clin Infect Dis. 2004;39(2):192-198.

14. Schaal JV, Leclerc T, Soler C, et al. Epidemiology of filamentous fungal infections in burned patients: a French retrospective study. Burns. 2015;41(4):853-863.

15. Marr KA, Balajee SA, McLaughlin L, Tabouret M, Bentsen C, Walsh TJ. Detection of galactomannan antigenemia by enzyme immunoassay for the diagnosis of invasive aspergillosis: variables that affect performance. J Infect Dis. 2004;190(3):641-649.

16. Chai LYA, Kullberg BJ, Johnson EM, et al. Early serum galactomannan trend as a predictor of outcome of invasive aspergillosis. J Clin Microbiol. 2012;50(7):2330-2336.
17. Boch T, Buchheidt D, Spiess B, Miethke T, Hofmann WK, Reinwald M. Direct comparison of galactomannan performance in concurrent serum and bronchoalveolar lavage samples in immunocompromised patients at risk for invasive pulmonary aspergillosis. Mycoses. 2016;59(2): 80-85.

18. Arvanitis M, Anagnostou T, Fuchs BB, Caliendo AM, Mylonakis E. Molecular and nonmolecular diagnostic methods for invasive fungal infections. Clin Microbiol Rev. 2014;27(3):490-526.

19. White PL, Wingard JR, Bretagne S, et al. Aspergillus polymerase chain reaction: systematic review of evidence for clinical use in comparison with antigen testing. Clin Infect Dis. 2015;61(8):1293-1303.

20. Walsh TJ, Anaissie EJ, Denning DW, et al; Infectious Diseases Society of America. Treatment of aspergillosis: clinical practice guidelines of the Infectious Diseases Society of America. Clin Infect Dis. 2008; 46(3):327-360.

21. Herbrecht R, Denning DW, Patterson TF, et al. Voriconazole versus amphotericin B for primary therapy of invasive aspergillosis. $N$ Engl $J$ Med. 2002;347(6):408-415.

22. Cornely OA, Maertens J, Bresnik M, et al. Liposomal amphotericin B as initial therapy for invasive mold infection: a randomized trial comparing a high-loading dose regimen with standard dosing (AmBiLoad trial). Clin Infect Dis. 2007;44(10):1289-1297.

23. Moriyama B, Obeng AO, Barbarino J, et al. Clinical pharmacogenetics implementation consortium (CPIC) guidelines for CYP2C19 and voriconazole dosing. Clin Pharmacol Ther. Epub 2016 Dec.

24. Marr KA, Schlamm HT, Herbrecht R, et al. Combination antifungal therapy for invasive aspergillosis a randomized trial. Ann Intern Med. 2015; 162(2):81-89.

25. Petraitis V, Petraitiene R, Hope WW, et al. Combination therapy in treatment of experimental pulmonary aspergillosis: in vitro and in vivo correlations of the concentration- and dose- dependent interactions between anidulafungin and voriconazole by bliss independence drug interaction analysis. Antimicrob Agents Chemother. 2009;53(6): 2382-2391.

26. Miceli MH, Kauffman CA. Isavuconazole: a new broad-spectrum triazole antifungal agent. Clin Infect Dis. 2015;61(10):1558-1565.

27. Rybak JM, Marx KR, Nishimoto AT, David Rogers P. Isavuconazole: pharmacology, pharmacodynamics, and current clinical experience with a new triazole antifungal agent. Pharmacotherapy. 2015;35(11):1037-1051.

28. Thompson GR, Wiederhold NP. Isavuconazole: a comprehensive review of spectrum of activity of a new triazole. Mycopathologia. 2010;170(5):291-313.

29. Schmitt-hoffmann A, Roos B, Heep M, et al. Multiple-dose pharmacokinetics and safety of the new antifungal triazole BAL4815 after intravenous infusion and oral administration of its prodrug, BAL8557, in healthy volunteers. Antimicrob Agents Chemother. 2006;50(1): 286-293.

30. Schmitt-Hoffmann A, Roos B, Heep M, et al. Single-ascending-dose pharmacokinetics and safety of the novel broad-spectrum antifungal triazole BAL4815 after intravenous infusions (50, 100, and 200 milligrams) and oral administrations (100, 200, and 400 milligrams) of its prodrug, BAL8557, in healthy volunteers. Antimicrob Agents Chemother. 2006;50(1):279-285.

31. Schmitt-Hoffmann A, Roos B, Spickermann J, et al. Effect of mild and moderate liver disease on the pharmacokinetics of isavuconazole after intravenous and oral administration of a single dose of the prodrug BAL8557. Antimicrob Agents Chemother. 2009;53(11): 4885-4890.

32. Desai A, Schmitt-hoffmann A, Mujais S. Population pharmacokinetics of isavuconazole in subjects with mild or moderate hepatic impairment. Antimicrob Agents Chemother. 2016;60(5):3025-3031.

33. Cornely OA, Bohme A, Schmitt-Hoffmann A, Ullmann AJ. Safety and pharmacokinetics of isavuconazole as antifungal prophylaxis in acute myeloid leukemia patients with neutropenia: results of a phase 2, dose escalation study. Antimicrob Agents Chemother. 2015;59(4): 2078-2085. 
34. Seyedmousavi S, Brüggemann RJM, Meis JF, Melchers WJG, Verweij PE, Moutona JW. Pharmacodynamics of isavuconazole in an Aspergillus fumigatus mouse infection model. Antimicrob Agents Chemother. 2015;59(5):2855-2866.

35. Astellas. Isavuconazonium: Invasive Aspergillosis and Invasive Mucormycosis. Anti-infective Drugs Advisory Committee Briefing Document; 2015 Jan:1-194.

36. Groll AH, Desai A, Han D, et al. Pharmacokinetic assessment of drugdrug interactions of isavuconazole with the immunosuppressants cyclosporine, mycophenolic acid, prednisolone, sirolimus, and tacrolimus in healthy adults. Clin Pharmacol Drug Dev. 2017;6(1):76-85.

37. de la Escalera CM, Aller AI, Lopez-Oviedo E, et al. Activity of BAL 4815 against filamentous fungi. J Antimicrob Chemother. 2008;61(5): 1083-1086.

38. Guinea J, Pelaez T, Recio S, Torres-Narbona M, Bouza E. In vitro antifungal activities of isavuconazole (BAL4815), voriconazole, and fluconazole against 1,007 isolates of Zygomycete, Candida, Aspergillus, Fusarium, and Scedosporium Species. Antimicrob Agents Chemother. 2008;52(4):1396-1400.

39. Rudramurthy SM, Chakrabarti A, Geertsen E, Mouton JW, Meis JF. In vitro activity of isavuconazole against 208 Aspergillus flavus isolates in comparison with 7 other antifungal agents: assessment according to the methodology of the European Committee on Antimicrobial Susceptibility Testing. Diagn Microbiol Infect Dis. 2011;71(4):370-377.

40. Warn PA, Sharp A, Denning DW. In vitro activity of a new triazole BAL4815, the active component of BAL8557 (the water-soluble prodrug), against Aspergillus spp. J Antimicrob Chemother. 2006;57(1):135-138.

41. Pfaller MA, Messer SA, Rhomberg PR, Jones RN, Castanheira M. In vitro activities of isavuconazole and comparator antifungal agents tested against a global collection of opportunistic yeasts and molds. J Clin Microbiol. 2013;51(8):2608-2616.

42. Perkhofer S, Lechner V, Lass-Florl C. In vitro activity of isavuconazole against Aspergillus species and zygomycetes according to the methodology of the European Committee on antimicrobial susceptibility testing. Antimicrob Agents Chemother. 2009;53(4):1645-1647.

43. Howard SJ, Lass-Florl C, Cuenca-Estrella M, Gomez-Lopez A, Arendrup MC. Determination of isavuconazole susceptibility of Aspergillus and Candida species by the EUCAST method. Antimicrob Agents Chemother. 2013;57(11):5426-5431.

44. Espinel-Ingroff A, Chowdhary A, Gonzalez GM, et al. Multicenter study of isavuconazole MIC distributions and epidemiological cutoff values for Aspergillus spp. for the CLSI M38-A2 broth microdilution method. Antimicrob Agents Chemother. 2013;57(8):3823-3828.
45. Arendrup MC, Meletiadis J, Mouton JW, et al. EUCAST technical note on isavuconazole breakpoints for Aspergillus, itraconazole breakpoints for Candida and updates for the antifungal susceptibility testing method documents. Clin Microbiol Infect. 2016;22(6):571.e1-571.e4.

46. Gregson L, Goodwin J, Johnson A, et al. In vitro susceptibility of Aspergillus fumigatus to isavuconazole: correlation with itraconazole, voriconazole, and posaconazole. Antimicrob Agents Chemother. 2013;57(11): $5778-5780$.

47. Lepak AJ, Marchillo K, VanHecker J, Andes DR. Isavuconazole (BAL4815) pharmacodynamic target determination in an in vivo murine model of invasive pulmonary aspergillosis against wild-type and cyp 51 mutant isolates of Aspergillus fumigatus. Antimicrob Agents Chemother. 2013;57(12):6284-6289.

48. Warn PA, Sharp A, Mosquera J, et al. Comparative in vivo activity of BAL4815, the active component of the prodrug BAL8557, in a neutropenic murine model of disseminated Aspergillus flavus. J Antimicrob Chemother. 2006;58:1198-1207.

49. Kovanda LL, Petraitiene R, Petraitis V, et al. Pharmacodynamics of isavuconazole in experimental invasive pulmonary aspergillosis: implications for clinical breakpoints. J Antimicrob Chemother. 2016;71(7): 1885-1891.

50. Petraitis V, Petraitiene R, Moradi PW, et al. Pharmacokinetics and concentration-dependent efficacy of isavuconazole for treatment of experimental invasive pulmonary aspergillosis. Antimicrob Agents Chemother. 2016;60(5):2718-2726.

51. Katragkou A, McCarthy M, Meletiadis J, et al. In vitro combination of isavuconazole with micafungin or amphotericin B deoxycholate against medically important molds. Antimicrob Agents Chemother. 2014;58(11):6934-6937.

52. Petraitis V, Petraitiene R, Zaw MH, et al. Synergistic activity of isavuconazole and micafungin: dosage optimization in treatment of experimental invasive pulmonary aspergillosis. Poster presented at: ASM Microbe; June 17; 2016; Boston, MA.

53. Maertens JA, Raad II, Marr KA, et al. Isavuconazole versus voriconazole for primary treatment of invasive mould disease caused by Aspergillus and other filamentous fungi (SECURE): a phase 3, randomisedcontrolled, non-inferiority trial. Lancet. 2015;6736(15):1-10.

54. de Pauw B, Walsh T, Donnelly J. Revised definitions of invasive fungal disease from the European Organization for research and treatment of cancer/invasive. Clin Infect Dis. 2008;46(12):1813-1821.

55. Marty FM, Ostrosky-Zeichner L, Cornely OA, et al. Isavuconazole treatment for mucormycosis: a single-arm open-label trial and casecontrol analysis. Lancet Infect Dis. 2016;3099(16):1-10.
Orphan Drugs: Research and Reviews

\section{Publish your work in this journal}

Orphan Drugs: Research and Reviews is an international, peer- reviewed open access journal publishing original research, reports, reviews and commentaries on all areas of the design and development of orphan drugs for the treatment of rare diseases through to clinical applications Clinical outcomes, patient safety, and programs for the development and

\section{Dovepress}

effective, safe, and sustained use of medicines will be a feature of the journal. The manuscript management system is completely online and includes a very quick and fair peer-review system, which is all easy to use. Visit http://www.dovepress.com/testimonials.php to read real quotes from published authors. 